\title{
Anemia of Inflammation (AI) Elevated Serum Hepcidin Level and Therapeutic Intravenous Administration of Iron
}

\author{
Kenji Shinohara, ${ }^{\mathrm{a}, \mathrm{b}}$
}

\begin{abstract}
An 81-year-old female complained of anorexia and puffy face. Complete blood cell counts (CBC) demonstrated normocytic normochromic anemia. Serum iron and total iron binding capacity (TIBC) were decreased, and ferritin was increased. CRP and acute phase proteins were elevated. Serum levels of interleukin (IL)-6 and hepcidin-25 were elevated. The patient was diagnosed as having anemia of inflammation (AI), although the cause was unknown. The oral administration of iron was ineffective, whereas intravenous administration was effective for the amelioration of anemia. The levels of ferritin and hepcidin increased, whereas those of CRP and IL-6 decreased without specific treatment.
\end{abstract}

Keywords: Anemia of inflammation; Interleukin-6; Hepcidin; Intravenous iron

\section{Introduction}

Anemia of chronic disease (ACD) is frequently seen in elderly patients with infections, inflammation, malignancies autoimmune disorders, and chronic renal and heart failure $[1,2]$, and anemia especially associated with inflammation is also termed as anemia of inflammation (AI). ACD/AI is typically characterized by a normocytic normochromic anemia, low serum iron, low transferrrin saturation, high ferritin, and blunted response to erythropoietin (EPO) $[1,2]$.

Hepcidin expression in the liver is induced by both iron overload and inflammatory stimuli [3-6]. Inflammatory cytokines such as interleukin (IL-6) released during infections or inflammatory diseases induce excess synthesis of hepcidin

\footnotetext{
Manuscript accepted for publication April 25, 2013

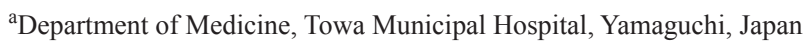

${ }^{\mathrm{b}}$ Corresponding address: 571-1, Suo-Oshima, Yamaguchi 742-2921,

Japan. Email: shinohara9642@suo-kouei.com

doi: http://dx.doi.org/10.4021/jmc1272w
}

[3-6]. Hepcidin controls transmembrane ferroportin activity of intestinal epithelial cells, inhibits iron export from the intestinal epithelium and from iron-recycling macrophages into blood plasma, and inhibits iron absorption from the intestine [3-6]. It restricts the availability of iron to microbes and increases the resistance to infections. Thus, hepcidin is the principal iron-regulatory hormone and is the key mediator of ACD/AI [3-6].

It was difficult to diagnose $\mathrm{ACD} / \mathrm{AI}$ until recently, since the assay of hepcidin was not easy, but now mass spectrometry and enzyme-linked immunosorbent assay (ELISA) methods are available $[1,2]$.

We report a case of AI, and the pathogenetic role of inflammatory reactions including elevated serum levels of acute phase proteins, IL- 6 and hepcidin, and therapeutic efficacy of intravenous administration of iron for amelioration of anemia.

\section{Case Report}

An 81-year-old female complained of anorexia and puffy face. The heart rate was $112 / \mathrm{min}$, with regular rhythm and no murmur. Liver and spleen were not palpable and ascites was not observed. Legs edema was not observed. Fever was not observed. Laboratory findings on admission one month later when anemia aggravated are shown in Table 1. Complete blood cell counts (CBC) demonstrated normocytic normochromic anemia. Dohle bodies were observed in the cytoplasm of neutrophils. Serum iron and total iron binding capacity (TIBC) were decreased, and ferritin was increased. Bone marrow aspiration smear from iliac bone demonstrated slightly hypocellular marrow, but abnormal cells or dysplastic cells were not observed. Chromosomal analysis of the bone marrow cells demonstrated 46,XX. Acute phase proteins including CRP, electrophoretic protein fractions, and $\mathrm{CH} 50$ were elevated. Rheumatoid factor (RF) was positive, however anti-cyclic citrullinated peptide antibody (anti-CCP $\mathrm{Ab}$ ) was negative. Clinical symptoms of rheumatoid arthritis (RA) were not observed.

Serum level of interleukin-6 (IL-6) was elevated, and erythropoietin (Epo) was normal. 
Table 1. Laboratory Data at and Around Admission



( ) normal values.

The predominant form of hepcidin in human contained 25 amino acids (hepcidin-25). Serum level of hepcidin-25 was measured by liquid chromatography and mass spectrometry using 4000QTRAP LC-MS/MS assay system (Applied Biosystems, Foster City, CA, USA) by Medical Care Proteomics Biotechnology Co., Ltd. (Ishikawa, Japan), was elevated.

AI of unknown cause was diagnosed. The clinical course is shown in Figure 1. Administration of iron orally, ferrous citrate sodium, $100 \mathrm{mg} / \mathrm{day}$, for one month, was ineffective for improving the hemoglobin concentration. The levels of $\mathrm{CRP}$ gradually dropped, however, the values of $\mathrm{Hb}$ were un- changed during next two months. Subsequently, intravenous administration of iron was performed, saccharated ferric oxide, $80 \mathrm{mg} /$ day once a week for 3 months, total amount of 960 $\mathrm{mg}$ according to the Wintrobe's formula for iron deficiency anemia (IDA), and it was effective for the amelioration of anemia. The increased levels of $\mathrm{Hb}$ were maintained for the last 4 months. Serum iron and ferritin were not increased during oral administration of iron, but they were increased during intravenous administration $(57 \mu \mathrm{g} / \mathrm{dL}$ and $779 \mathrm{ng} / \mathrm{mL}$, respectively), and that of hepcidin increased after intravenous administration of iron and amelioration of anemia.

The levels of CRP and IL-6 decreased without specific 


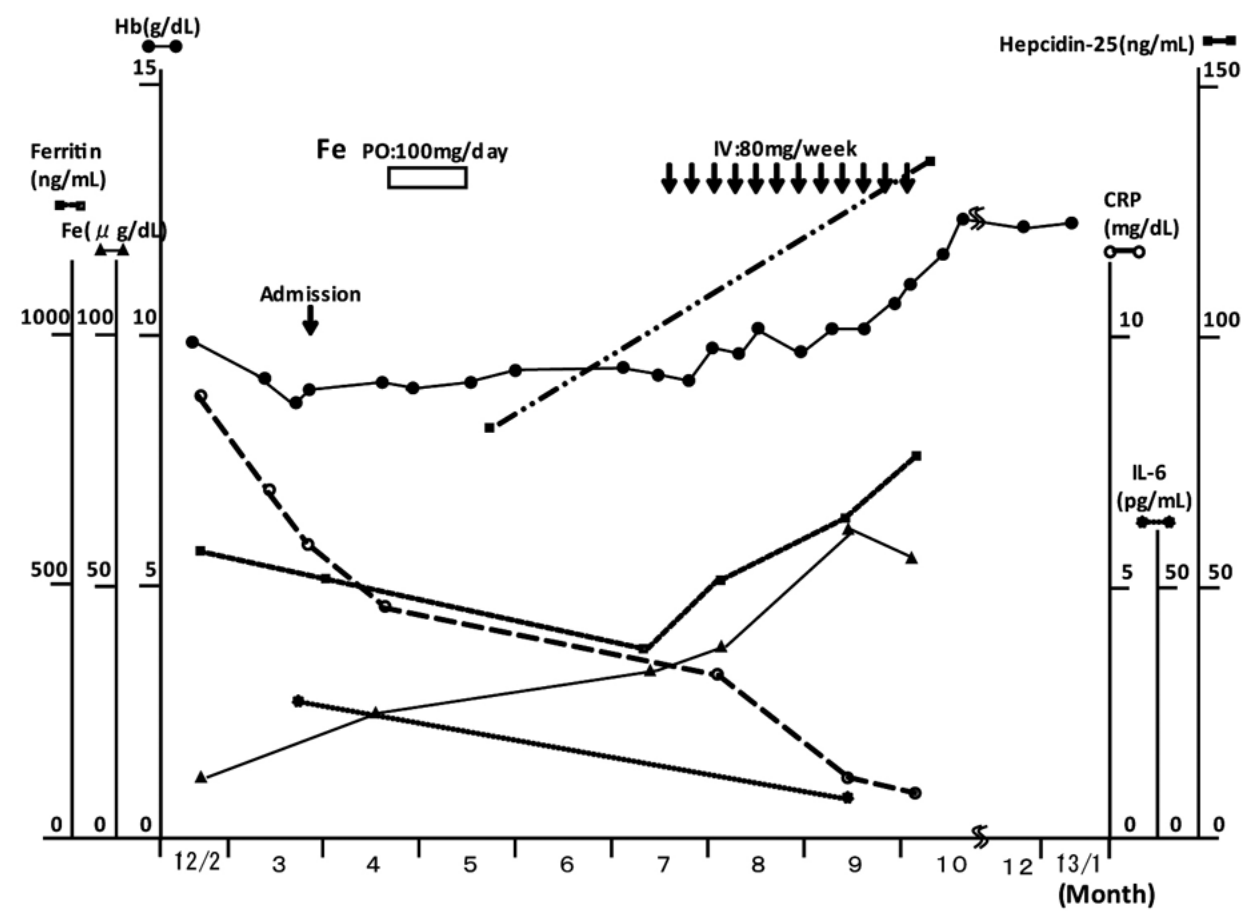

Figure 1. Clinical course.

treatment.

\section{Discussion}

In the present patient, the inflammatory process as evidenced by the increased of acute phase proteins including IL-6 may be present. However, the cause of inflammation was not clear. In the present patient, RA was not considered, since although RF was positive, however RA specific anti-CCP Ab was negative, and clinical symptom of RA was not observed. Infections, cancer and autoimmune disorders, and Castleman disease were also not considered in the present patient.

AI in noninflammatory or mildly inflammatory states may be present in the elderly; anemia of heart failure, and anemia of chronic kidney disease (CKD) $[1,2]$. In the patients with heart failure, $57 \%$ of them had AI, and had higher levels of inflammatory markers including IL-6 and tumor necrosis factor (TNF)- $\alpha$, and blunted Epo production and defective iron supply for hematopiesis [7]. The pathogenesis of anemia in CKD is predominantly driven by impaired Epo production. In addition, the serum levels of hepcidin, ferritin and IL-6 were elevated, and low grade inflammation decreased the avalability of iron for hematopoiesis [8].

In patients with RA, the roles of IL-6 and hepcidin were considered for the pathogenesis of ACD/AI [9].

Differentiation of true iron deficiency anemia (IDA) in AI is important [10]. The ratio of serum transferrin receptor (sTfR) to ferritin ( $\mathrm{R} / \mathrm{F}$ ratio) has been shown to estimate body iron stores, but it cannot be used widely for the lack of standardization of sTfR assays [10]. Serum hepcidin levels were significantly higher in patients with inflammation alone than those with either IDA alone or combined IDA and inflammation [10]. In the present patient, true IDA may not be complicated but functional iron deficiency might be present since red blood cells were normocytic and normochromic, TIBC was decreased, ferritin was increased, whereas unsaturated iron binding capacity (UIBC) was normal, and the level of hepcidin at admission was extremely high.

The serum ferritin levels are increased in both iron overload and inflammation [10]. Bone marrow aspiration smear stained with Prussian blue is considered the most reliable method to determine bone marrow iron stores, however this was not performed in the present patient. In the present patient, it is reasonable to consider that the increased serum hepcidin by inflammation may inhibit iron absorption from the intestine and also inhibit the release of iron from macrophages, resulting in decrease of serum iron and increase in the ferritin levels.

From the standpoint of therapy in ACD/AI, basic management of the causative disease process will usually improve anemia $[1,2]$.

In the present patient, the levels of CRP and IL- 6 decreased during clinical course without specific treatment. Amelioration of inflammation might also contribute to the amelioration of anemia.

In the present patient, oral iron administration for one month was ineffective for amelioration of anemia. After in- 
flammation disappeared, the lowering of hepcidin-25 level and the improved utilization of stored iron might be expected. This remained unanswered in the present patient, since the level of hepcidin-25 was not measured, and $\mathrm{Hb}$ concentrations during these months after oral iron administration, were not increased.

There are several reports that supplemental iron given intravenously can safely ameliorate anemia of functional iron deficiency in ACD/AI [2, 11]. The favorable effects were observed mostly in the patients with anemia of dialysis, cancer and cancer chemotherapy, and amelioration of anemia in chronically ill patients improved quality of life and survival $[12,13]$. There are several intravenous (IV) iron preparations, and after injections, they are mostly phagocytosed in the reticuloendothelial system $[12,13]$. In the dialysis patients' response to IV iron with elevated ferritin (DRIVE) trial, it was effective in the patients who had serum ferritin above $500 \mathrm{ng} / \mathrm{mL}$, and as high as 1,200 ng/mL, with concurrent transferrin saturation below $25 \%$, and CRP levels above the normal range [12]. The study concluded that IV iron is effective in overcoming inflammation-mediated reticuloendothelial blockade of iron [12]. Although the degree of anemia was moderate in the present patient, intravenous administration of iron was performed for further increase of $\mathrm{Hb}$ concentration and quality of life. IV iron quickly increased $\mathrm{Hb}$ level during administration, it was maintained for the last 4 months after administration, and the patient felt well-being. Oral iron administration did not increase the serum levels of iron, whereas IV iron increased the levels of serum iron and the levels of ferritin were further increased. These results indicated that intravenous administration of iron was primarily effective for the amelioration of anemia in the present patient, and suggested that intravenously administrated iron might be partly mobilized into the serum and utilized for hemoglobin synthesis.

Acute adverse events of anaphylactoid reactions were not observed in the present patient; however, excess iron intravenous administration must be avoided because of the deleterious effects. In the present patient, the intravenous administration of iron was performed according to the Wintrobe's formula for IDA, and the total dose of intravenously administrated iron was within the calculated dose, and the administration was stopped shortly after anemia was ameliorated. The level of hepcidin increased after intravenous administration of iron and amelioration of anemia, and this may be due to the increased body iron by intravenous administration of iron. It is unknown whether the efficacy of intravenous administration of iron is temporary, and maintenance IV iron therapy is necessary. The long-term observation of the efficacy and safety of intravenous administration of iron such as iron overload and organ damage, should be performed.

Alternative therapies that target hepcidin-ferroportin axis (direct hepcidin antagonists, hepcidin production inhibitors, ferroportin antagonists/stabilizers), have been devel- oped as the new treatment strategies for ACD/AI [14].

Considering the role of pro-inflammatory cytokine IL-6 in the pathogenesis of AI, IL-6 represents an important target in AI $[9,15]$. Treatment with anti-IL-6 receptor antibody (tocilizumab) reduced serum hepcidin levels and improved AI in Castleman disease [15]. These treatments should be considered in the near future.

The number of such patients as the present case should not be underestimated, since such patients may increase after the understanding of the pathophysiology of $\mathrm{ACD} / \mathrm{AI}$ in the era of aging society. The assay of serum hepcidin activity should be performed frequently to diagnose ACD/AI.

\section{Acknowledgement}

The author thanks professor Yutaka Kogo and lecturer Katsuya Imada of Asahikawa Medical School, Third Department of Internal Medicine, for consulting and the kind advice on the present patient.

\section{Disclosure}

The author reported no conflict of interest. Written permission has been obtained from the patient to write this report. This Report has never been published in any other journal.

\section{References}

1. Guralnik JM, Ershler WB, Schrier SL, Picozzi VJ. Anemia in the elderly: a public health crisis in hematology. Hematology Am Soc Hematol Educ Program. 2005:528532.

2. Cullis JO. Diagnosis and management of anaemia of chronic disease: current status. Br J Haematol. 2011;154(3):289300.

3. Ganz T. Hepcidin, a key regulator of iron metabolism and mediator of anemia of inflammation. Blood. 2003;102(3):783-788.

4. Roy CN, Andrews NC. Anemia of inflammation: the hepcidin link. Curr Opin Hematol. 2005;12(2):107-111.

5. Ganz T. Hepcidin and iron regulation, 10 years later. Blood. 2011;117(17):4425-4433.

6. Andrews NC. Closing the iron gate. N Engl J Med. 2012;366(4):376-377.

7. Opasich C, Cazzola M, Scelsi L, De Feo S, Bosimini E, Lagioia R, Febo O, et al. Blunted erythropoietin production and defective iron supply for erythropoiesis as major causes of anaemia in patients with chronic heart failure. Eur Heart J. 2005;26(21):2232-2237.

8. Tomosugi N, Kawabata H, Wakatabe R, Higuchi M, Yamaya H, Umehara H, Ishikawa I. Detection of serum 
hepcidin in renal failure and inflammation by using ProteinChip System. Blood. 2006;108(4):1381-1387.

9. Raj DS. Role of interleukin-6 in the anemia of chronic disease. Semin Arthritis Rheum. 2009;38(5):382-388.

10. Theurl I, Aigner E, Theurl M, Nairz M, Seifert M, Schroll A, Sonnweber T, et al. Regulation of iron homeostasis in anemia of chronic disease and iron deficiency anemia: diagnostic and therapeutic implications. Blood. 2009;113(21):5277-5286.

11. Goodnough LT, Nemeth E, Ganz T. Detection, evaluation, and management of iron-restricted erythropoiesis. Blood. 2010;116(23):4754-4761.

12. Coyne DW, Kapoian T, Suki W, Singh AK, Moran JE, Dahl NV, Rizkala AR. Ferric gluconate is highly efficacious in anemic hemodialysis patients with high serum ferritin and low transferrin saturation: results of the Di- alysis Patients' Response to IV Iron with Elevated Ferritin (DRIVE) Study. J Am Soc Nephrol. 2007;18(3):975984.

13. Auerbach M, Coyne D, Ballard H. Intravenous iron: from anathema to standard of care. Am J Hematol. 2008;83(7):580-588.

14. Sun CC, Vaja V, Babitt JL, Lin HY. Targeting the hepcidin-ferroportin axis to develop new treatment strategies for anemia of chronic disease and anemia of inflammation. Am J Hematol. 2012;87(4):392-400.

15. Song SN, Tomosugi N, Kawabata H, Ishikawa T, Nishikawa T, Yoshizaki K. Down-regulation of hepcidin resulting from long-term treatment with an anti-IL-6 receptor antibody (tocilizumab) improves anemia of inflammation in multicentric Castleman disease. Blood. 2010;116(18):3627-3634. 